\title{
THU0222 B CELL MODULATORY VARIANT TNF RECEPTOR DOMAINS (VTDS) IDENTIFIED BY DIRECTED EVOLUTION TO INHIBIT BAFF AND APRIL, ALONE OR COMBINED WITH VARIANT IG DOMAINS (VIGD'M) THAT INHIBIT T CELL COSTIMULATION, FOR THE TREATMENT OF SEVERE AUTOIMMUNE AND/OR INFLAMMATORY DISEASE
}

S. R. Dillon ${ }^{1}$, L. S. Evans ${ }^{2}$, M. W. Rixon ${ }^{3}$, J. Kuijper ${ }^{4}$, D. Demonte ${ }^{5}$, K. E. Lewis ${ }^{6}$, S. Levin ${ }^{2}$, K. Kleist6, S. Mudri' ${ }^{6}$, S. Bort ${ }^{2}$, J. Bhandari ${ }^{3}$, F. Ahmed-Qadri ${ }^{6}$ J. Yang ${ }^{7}$, M. A. Seaberg ${ }^{8}$, R. Wang ${ }^{8}$, R. Sanderson ${ }^{8}$, M. F. Wolfson ${ }^{3}$, J. Hillson ${ }^{7}$, S. L. Peng ${ }^{7}$, K. M. Swiderek ${ }^{9}{ }^{1}$ Alpine Immune Sciences Inc, Translational Sciences, Seattle, United States of America; ${ }^{2}$ Alpine Immune Sciences Inc, Immunology, Seattle, United States of America; ${ }^{3}$ Alpine Immune Sciences Inc, Protein Therapeutics, Seattle, United States of America; ${ }^{4}$ Alpine Immune Sciences Inc, Protein Engineering, Seattle, United States of America; ${ }^{4} A / p i n e$ Immune Sciences Inc, Protein Engineering, Seattle, United States of America; ${ }^{1}$ Alpine Immune Sciences Inc, Translational Sciences, Seattle, United States of America; ${ }^{7}$ Alpine Immune Sciences Inc, Clinical Development, Seattle, United States of America; ${ }^{8}$ Alpine Immune Sciences Inc, Bioanalytical Sciences, Seattle, United States of America; ${ }^{9}$ Alpine Immune Sciences Inc, Research, Seattle, United States of America

Background: BAFF and APRIL are TNF superfamily members that bind both TACI and BCMA on B cells; BAFF also binds BAFF-R. Together, BAFF and APRIL support $B$ cell development, differentiation, and survival. Their co-neutralization dramatically reduces $B$ cell function, including antibody production, whereas inhibition of either BAFF or APRIL alone mediates relatively modest effects.

Objectives: While CTLA-4-based therapeutics that block T cell costimulation provide safe and moderately effective $T$ cell inhibition in many disease settings, and while B cell targeting therapies have demonstrated promising therapeutic potential, we postulate that improved, combined BAFF and APRIL inhibition, either alone or coupled with inhibition of $\mathrm{T}$ cell costimulation, will provide more effective and durable relief from severe B cell-related autoimmune diseases like SLE.

Methods: We used our directed evolution platform to identify variant domains of the TNF family receptors TACI or BCMA that exhibit enhanced affinity for BAFF and APRIL as compared to their wild-type (WT) counterparts. These variant TACI or BCMA domains (VTD), alone or together with platform-derived CTLA-4 domains (vlgD), were fused to a modified human IgG1 Fc lacking effector function, yielding a panel of immunomodulatory molecules: TACI vTD-Fc, BCMA vTD-Fc, TACI vTD/ CTLA-4 vlgD-Fc, \& BCMA vTD/CTLA-4 vlgD-Fc. All were evaluated for functional activity: 1) in vitro in primary human $B$ cell \& MLR assays and in a Jurkat/NF-kB reporter cell line expressing $\mathrm{TACl}$, and 2) in vivo in standard immunization models, and in the bm12-induced and NZB/NZW spontaneous mouse models of lupus.

Results: The novel engineered TACI VTD-FC or BCMA vTD-Fc fusion proteins significantly inhibited BAFF- and APRIL-mediated signaling in vitro in $\mathrm{TACl}^{+}$Jurkat cells. TACl (or BCMA) vTD/CTLA-4 vlgD-Fc proteins also attenuated T cell activation in primary human lymphocyte assays. When administered to mice, these molecules rapidly and potently reduced key $B$ and $T$ cell subsets, including plasma cells, follicular T helper cells, germinal center cells, \& memory T cells. Treatment with TACI vTD-Fc or TACI vTD/CTLA-4 vlgD-Fc proteins also significantly reduced titers of antigen-specific antibodies in immunized mice more so than abatacept or WT TACI-Fc, and potently suppressed anti-dsDNA autoantibodies, blood urea nitrogen levels, proteinuria, and renal immune complex deposition in the bm12 \& NZB/W lupus models.

Conclusion: Directed evolution of TNFR and IgSF domains has successfully facilitated the development of $\mathrm{FC}$ fusion proteins containing TACI or BCMA vTDs, with or without fusion to CTLA-4 vlgDs. These novel immunomodulators consistently demonstrate potent immunosuppressive activity and efficacy in vitro and in vivo, appearing superior to existing and/or approved immunomodulators like belimumab, abatacept, or atacicept. Such biologics may therefore be attractive candidates for the treatment of serious autoimmune diseases, particularly B cell-related diseases such as SLE, Sjogren's syndrome, etc.

NZB/W: Proteinuria Scores

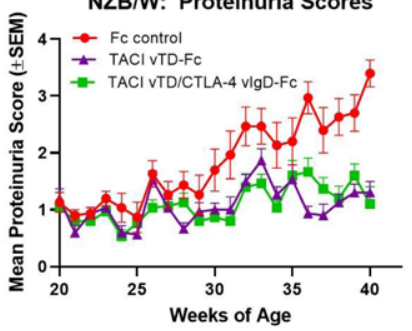

NZB/W: Serum [anti-dsDNA]

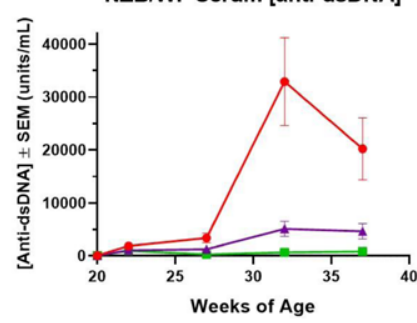

Disclosure of Interests: : Stacey R. Dillon Shareholder of: Shareholder of Alpine Immune Sciences, Inc., Employee of: Employee of Alpine Immune Sciences, Inc. Lawrence S. Evans Shareholder of: Shareholder of Alpine Immune Sciences, Inc., Employee of: Employee of Alpine Immune Sciences, Inc., Mark W. Rixon Shareholder of: Shareholder of Alpine Immune Sciences, Inc., Employee of: Employee of Alpine Immune Sciences, Inc., Joe Kuijper Shareholder of: Shareholder of Alpine Immune Sciences, Inc., Employee of: Employee of Alpine Immune Sciences, Inc., Dan Demonte Shareholder of: Shareholder of Alpine Immune Sciences, Inc., Employee of: Employee of Alpine Immune Sciences, Inc., Katherine E. Lewis Shareholder of: Shareholder of Alpine Immune Sciences, Inc., Employee of: Employee of Alpine Immune Sciences, Inc., Steve Levin Shareholder of: Shareholder of Alpine Immune Sciences, Inc. Employee of: Employee of Alpine Immune Sciences, Inc., Kayla Kleist Shareholder of: Shareholder of Alpine Immune Sciences, Inc., Employee of: Employee of Alpine Immune Sciences, Inc., Sherri Mudri Shareholder of: Shareholder of Alpine Immune Sciences, Inc., Employee of: Employee of Alpine Immune Sciences, Inc., Susan Bor Shareholder of: Shareholder of Alpine Immune Sciences, Inc., Employee of: Employee of Alpine Immune Sciences, Inc., Janhavi Bhandari Shareholder of: Shareholder of Alpine Immune Sciences, Inc., Employee of: Employee of Alpine Immune Sciences, Inc., Fariha Ahmed-Qadri Shareholder of: Shareholder of Alpine Immune Sciences, Inc., Employee of: Employee of Alpine Immune Sciences, Inc., Jing Yang Shareholder of: Alpine Immune Sciences, Inc., Employee of: Alpine Immune Sciences, Inc. Michelle A. Seaberg Shareholder of: Shareholder of Alpine Immune Sciences, Inc., Employee of: Employee of Alpine Immune Sciences, Inc., Rachel Wang Shareholder of: Shareholder of Alpine Immune Sciences, Inc., Employee of: Employee of Alpine Immune Sciences, Inc., Russell Sanderson Shareholder of: Alpine Immune Sciences, Inc., Employee of: Alpine Immune Sciences, Inc., Martin F. Wolfson Shareholder of: Shareholder of Alpine Immune Sciences, Inc., Employee of: Employee of Alpine Immune Sciences, Inc., Jan Hillson Shareholder of: Alpine Immune Sciences, Inc. Employee of: Alpine Immune Sciences, Inc., Stanford L. Peng Shareholder of: Alpine Immune Sciences, Inc., Employee of: $\mathrm{CMO}$ and President of Alpine Immune Sciences, Inc., Kristine M. Swiderek Shareholder of: Shareholder of Alpine Immune Sciences, Inc., Employee of: Employee of Alpine Immune Sciences, Inc. DOI: 10.1136/annrheumdis-2020-eular. 1545

\section{THU0223 \\ THE NEUROPSYCHIATRIC PHENOTYPE OF NZB/W LUPUS-PRONE MOUSE MODEL AT PRE-NEPHRITIC AND NEPHRITIC STAGES OF THE DISEASE: MURINE MODEL RECAPITULATES HUMAN DISEASE}

D. Nikolopoulos ${ }^{1,2}$, A. Polissidis ${ }^{2}$, K. Karali ${ }^{2}$, D. Boumpas ${ }^{1,2} \cdot{ }^{1}$ National and Kapodistrian University of Athens, Medical School, 4th Department of Internal Medicine, "Attikon" University Hospital, Athens, Greece; ${ }^{2}$ Biomedical Research Foundation Academy of Athens, Athina, Greece

Background: The pathogenesis of neuropsychiatric lupus (NPSLE) remains ill-defined due to limited access to tissue and the diversity and complexity of clinical manifestations with most evidence deriving from animal studies in MLR/ Ipr lupus-prone mouse.

Objectives: To phenotype and characterize the neuropsychiatric disease in New Zealand Black/White (NZB/W) F1 strain; a spontaneous lupus prone mouse which is characterized by systemic autoimmunity.

Methods: Mice were tested in a comprehensive behavioral test battery to assess possible effects on general depressive-like disorders, anxiety, cognitive function and motor performance/coordination. The following tests were conducted in the same order for all subjects (All females, NZB/W strain $n=13$ and C57BL/6 $n=14$ ) at 3 and 6 months of age: Open field, Novel object recognition (NOR), Novel object location (NOL), Elevated plus maze (EPM), Rotarod, Tail suspension test (TST), Prepulse inhibition (PPI), and Sucrose preference test (SPT). For comparisons, statistical significance was indicated as a two-sided $\mathrm{P}<0.05$.

Elevated plus maze

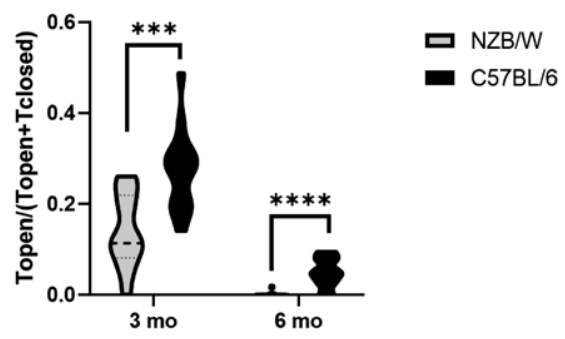

Graph 1. Elevated plus maze (EPM) evaluates anxiety-like phenotype. NZB/W mice exhibit anxiety-like phenotype at 3 months of age ( $P<0.001$, unpaired t-test) and at 6 months of age ( $P<0.0001$, unpaired t-test). NZB/ $/$ mice become months of age ( $P<0.001$, unpaired $t$-test) and at 6 months of age ( $P<0.0001$, unp $)$ 
Results: NZB/W mice at 3 months and 6 months of age exhibit depressive-like disorder as assessed by SPT and TST ( $P<0.05$ and $<0.0001$, respectively). Anxiety-like phenotype was evident in lupus-prone mice at both time points based on EPM test (Graph 1). Open-field test revealed decreased locomotor activity and rotarod (Graph 2) showed impaired motor coordination in 3 month-old and 6 month-old NZB/W mice ( $\mathrm{P}<0.001$ and $<0.01$, respectively). NZB/W mice exhibit cognitive dysfunction at 3 and 6 months of age based on NOR test $(P<0.05)$. No differences in cognitive function was observed between the two groups $(P=0.11)$. Prepulse inhibition test revealed decreased sensorimotor gating in 3 month-old NZB/W mice, a difference not reaching statistical significance $(P=.078)$. It was not possible to interpret correctly the PPI at second time point (6 months of age) due to age-related hearing loss in B6 at 6 month-old. NZB/W become more anxious over the course of the disease as assessed by EPM ( 3 mo. versus 6 mo. $\mathrm{P}<0.001$, paired t-test, Graph 1).

Conclusion: The NZB/W lupus-prone strain exhibit depressive-like behavior, anxiety, cognitive impairment and motor disturbances both at early and late stages of the disease. This polygenic murine model may be more suitable for investigating the autoimmunity-mediated neuroinflammation in human SLE.

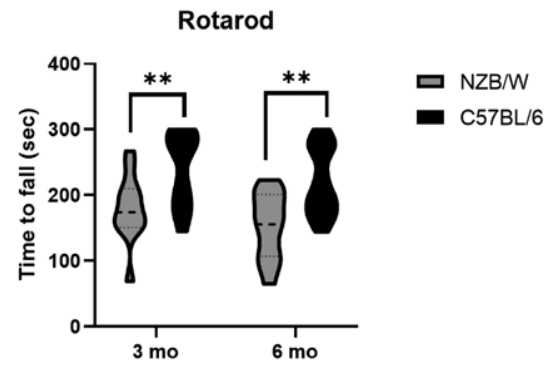

Graph 2. Rotarod performance
age $(P<0.01$, unpaired t-test)

Disclosure of Interests: : None declared

DOI: 10.1136/annrheumdis-2020-eular.1807

\section{\begin{tabular}{|l|l}
\hline THU0224 SKIN PROTEOME INVESTIGATION IN CUTANEOUS \\
\hline
\end{tabular} LUPUS ERYTHEMATOSUS (CLE) REVEALS NOVEL UNIQUE DISEASE PATHWAYS}

T. Niewold ${ }^{1}$, K. Popovic-Silwerfeldt ${ }^{2}$, J. Lehman ${ }^{3}$, A. Meves4, C. Charlesworth ${ }^{5}$, B. Madden ${ }^{5}$, A. Hayry ${ }^{6}$, A. Antovic ${ }^{6}$, I. E. Lundberg ${ }^{6}$, M. Wahren-Herlenius ${ }^{6}$, E. Svenungsson ${ }^{6}, \underline{\text { V. Oke }}{ }^{6} .{ }^{1}$ Colton Center for Autoimmunity, New York University, New York, United States of America; ${ }^{2}$ Karolinska Institutet, Institution for Clinical Sciences at Danderyd Hospital, Stockholm, Sweden; ${ }^{3}$ Mayo Clinic, Dermatology, Rochester, United States of America; ${ }^{3}$ Mayo Clinic, Dermatology, Rochester, United States of America; ${ }^{5}$ Mayo Clinic, Proteomics Core, Rochester, United States of America; ${ }^{6}$ Karolinska Institutet, Dept of Medicine, Stockholm, Sweden

Background: Cutaneous lupus erythematosus (CLE) is an autoimmune disease. It can be limited to the skin or be one of manifestations of systemic LE (SLE). The typical histopathologic pattern in CLE/SLE is interface dermatitis, which can also be observed in dermatomyositis (DM). While LE may affect any organ system, DM most commonly affect muscles and skin

Objectives: The aim of this study was to investigate the whole proteome of skin inflammatory foci in the cohort of CLE and DM patients in a comparatory, hypothesis-free manner and identify disease-unique molecular mechanisms.

Methods: CLE $(n=6)$, DM $(n=5)$ patients and controls $(n=6)$ were recruited at diagnosis or disease exacerbation. Skin biopsies were acquired, examined by a pathologist and selected inflammatory foci were laser micro-dissected. The total protein content was analyzed by mass-spectrometry, further analysis was performed by string-db.org platform. Certain proteomic findings were confirmed by immunohistochemistry (IHC).

Results: CLE infiltrates were more protein rich in comparison to DM lesions. There ratio of $5 x$ upregulated proteins in LE/DM was 60 , while ratio for DM/ LE was 13. Our results confirmed high abundance of (IFN)-regulated proteins both in CLE and DM, including: IFIT, MX and OAS families. Proteins expressed differentially in CLE covered complement proteins $(C 1 b)$, including membrane attack complex (MAC) $(\mathrm{C} 5, \mathrm{C} 6, \mathrm{C} 7, \mathrm{C} 8 \mathrm{~A}$ and $\mathrm{B})$ and complement regulators (CFHR1, CFHR2, CFHR5), as well as regulators of coagulation: thrombospondin 2 (THBS2), thrombin (F2), fibrinogen (F12) and annexin A3 (ANXA3). Importantly, we identified interleukin (IL) -16 as the only detectable and highly abundant cytokine in the CLE lesions and confirmed this finding by $\mathrm{IHC}$.

Conclusion: Conclusions Our data confirm evidence on IFN-regulated processes in CLE/SLE. Importantly, we identified IL-16 as a novel cytokine most strongly upregulated locally in the skin lesions. Moreover, we identified activation of MAC, complement regulating proteins as well as involvement of coagulation/fibrinolysis system. The study brings information on novel pathways involved in the inflammatory foci of the skin lesions in CLE patients. Our findings are of interest in further search of new therapeutic targets.

Disclosure of Interests: : Timothy Niewold: None declared, Karin Popovic-Silwerfeldt: None declared, Julia Lehman: None declared, Alexander Meves: None declared, Cristine Charlesworth: None declared, Benjamin Madden: None declared, Aliisa Hayry: None declared, Aleksandra Antovic: None declared, Ingrid E. Lundberg Grant/research support from: Bristol Meyer Squibb, Corbus Pharmaceuticals, Inc and Astra Zeneca, Marie Wahren-Herlenius: None declared, Elisabet Svenungsson: None declared, Vilija Oke: None declared

DOI: 10.1136/annrheumdis-2020-eular.5948

\begin{tabular}{|l|l}
\hline THU0225 & INTEGRATIVE PLASMA METABOLOME AND \\
TRANSCRIPTOME ANALYSIS REVEALED THE \\
IMPORTANCE OF HISTIDINE HOMEOSTASIS IN SLE \\
PATHOGENESIS WITH POTENTIAL FOR IMPROVED \\
SLE PATIENTS STRATIFICATION
\end{tabular}

Y. Iwasaki ${ }^{1}$, Y. Takeshima ${ }^{2}$, M. Nakano ${ }^{1}$, M. Ota ${ }^{3}$, Y. Nagafuchi ${ }^{3}$, A. Suzuki ${ }^{4}$, Y. Kochi ${ }^{5}$, T. Okamura ${ }^{5}$, T. Endo ${ }^{6}$, I. Miki ${ }^{6}$, K. Sakurada ${ }^{6}$, K. Yamamoto ${ }^{4}$ K. Fujio ${ }^{1}$. 'Graduate School of Medicine, The University of Tokyo, Allergy and Rheumatology, Tokyo, Japan; ${ }^{2}$ Graduate School of Medicine, The University of Tokyo, Functional Genomics and Immunological Diseases, Tokyo, Japan; ${ }^{3}$ Graduate School of Medicine, The University of Tokyo, Functional Genomics and Immunological Diseases, Tokyo, Japan; ${ }^{4}$ Center for Integrative Medical Sciences, RIKEN, Yokohama, Laboratory for Autoimmune Diseases, Yokohama, Japan; ${ }^{5}$ Tokyo Medical and Dental University, Genomic Function and Diversity, Tokyo, Japan; ${ }^{6}$ Cluster for Science, Technology Innovation Hub, RIKEN, Medical Sciences Innovation Hub Program, Yokohama, Japan

Background: Recently, immunometabolism has gathered attention of many immunologists. It has been widely recognized that metabolic reprogramming in each immune cell brings different effects on different cells and is important for regulating their functions. Along with the progress of statistical genetics, serum metabolites were shown to be under genetic regulations1). Metabolic changes are now considered not only to be mere phenotypes of cells but also to be key factors for controlling immune cell differentiation, proliferation and function through regulating gene expressions eventually. Although genome-wide association studies have brought deep insights into SLE pathogenesis, the precise pathway from genome to metabolome has been largely unknown, and vice versa.

Objectives: The aim of this study is to investigate metabolomic regulation in SLE in relation to gene expressions by integrating plasma metabolome data and transcriptome data.

Methods: We collected plasma samples from patients with SLE $(n=57)$ who met the 1997 American College of Rheumatology criteria for SLE. Gender- and age-matched healthy controls $(\mathrm{HCs})(\mathrm{n}=56)$ were recruited. Metabolic profiles focusing on 39 amino acids were analyzed with liquid chromatography (LC)mass spectrometry. Transcriptome data of SLE patients were obtained from our RNA-sequencing data of each immune cell subset (total 19 subsets). Whole-genome sequencing was also performed.

Results: Our previous experiment showed that about 160 peaks were detected from comprehensive LC-TOFMS and amino acids were useful for distinguishing SLE patients from HCs. Both partial least squares discriminant analysis (PLS-DA) and random forest, a machine learning algorithm, revealed the importance of histidine (His), one of the essential amino acids, to classify SLE patients from HCs, whose plasma level was lower in SLE patients. In addition, inverse correlation between His level and titer of ds-DNA as well as damage index (SDI) was detected. His level was correlated neither with PSL dosage nor with type I interferon (IFN) signature. Receiver operating characteristic (ROC) analysis showed the best predictability for SLE with the combination of specific amino acids including His. Our transcriptome analysis has revealed the significance of oxidative phosphorylation (OXPHOS) in B cells for SLE pathogenesis. Interestingly, OXPHOS signature was inversely correlated with His level in SLE B cells. Conclusion: His may be an important factor for SLE pathogenesis especially in B cells independently from IFN signal. SLC15A4, a transporter of His on lysosome, is one of the SLE GWAS SNPS and has been reported to play an 